\title{
Perspective
}

PERSPECTIVE Actualité en histoire de l'art

1 | 2018

Actualité en histoire de l'art

\section{La valeur des images}

\section{Martine Denoyelle}

\section{(2) OpenEdition}

Journals

Édition électronique

URL : http://journals.openedition.org/perspective/10103

DOI : 10.4000/perspective.10103

ISSN : 2269-7721

Éditeur

Institut national d'histoire de l'art

Édition imprimée

Date de publication : 30 juin 2018

Pagination : 183-186

ISBN : 978-2-917902-46-2

ISSN : $1777-7852$

Référence électronique

Martine Denoyelle, "La valeur des images », Perspective [En ligne], 1 | 2018, mis en ligne le 31

décembre 2018, consulté le 01 octobre 2020. URL : http://journals.openedition.org/perspective/10103 ; DOI : https://doi.org/10.4000/perspective.10103 


\title{
POSTFACE
}

\section{La valeur des images}

\author{
Martine Denoyelle
}

Dans l'éditorial du numéro 2012-1 de Perspective, Peter Weibel pointait la mutation fondamentale des images provoquée par leur usage grandissant sur internet ${ }^{1}$ et la métamorphose de l'usager de "destinataire " en "destinateur ", devenu producteur et distributeur de la représentation. Six ans plus tard, le constat de l'auteur appelle une autre réflexion : à quoi la dissolution du pouvoir des images dans les usages du web a-t-elle cédé la place ? Comment se recomposent les attributs symboliques et fonctionnels des images, et quelles sont les incidences de cette évolution sur leur statut, notamment celui des représentations digitales d'œuvres d'art et d'artefacts, qui alimentent la majeure partie des activités liées à leur connaissance ${ }^{2}$ ?

Les six années qui se sont écoulées depuis ont marqué une accélération considérable dans le développement de nouvelles productions et de nouveaux outils, ce à travers deux grands types d'initiatives : les régimes de libre réutilisation des images exemptes de droits et la diffusion d'outils numériques de gestion, de publication et d'interopérabilité des images ${ }^{3}$. Si le phénomène naît sous l'impulsion d'acteurs sociaux, un certain nombre d'institutions culturelles et de recherche y ont depuis adhéré, affirmant ainsi leur présence sur le web et la mise en place d'une nouvelle forme d'autorité, qui n'est plus liée à la détention de l'information textuelle ou visuelle, mais à la garantie qu'elles fournissent sur leur origine et sur leur qualité.

Au cour de ces propositions se tient aussi une réflexion approfondie sur les divers registres de valeurs portés par les images : valeur commerciale (constituante, par exemple de l'économie des organismes détenteurs de collections), valeur documentaire, valeur d'usage ou valeur de partage. Ces notions relèvent de catégories distinctes et superposables, que la place occupée aujourd'hui par le numérique tend à transformer en profondeur et, dans certains cas même, à remettre en question. Si l'on assiste bien, comme le relevait Peter Weibel, à une redistribution du pouvoir de l'image, une relation inédite entre économie, savoir et culture se fait surtout jour, née de l'identification de nouveaux besoins et des bénéfices potentiels d'un "engagement digital " de la part des institutions ${ }^{4}$. Les avantages ne relèvent plus, aujourd'hui, de la simple prospective : au-delà de l'effet d'annonce qui permet à ceux qui s'y engagent de se doter d'une identité dynamique ou pionnière ${ }^{5}$, l'adoption par les musées des politiques d'Open Access et d'Open Content commence, depuis quelques années, à pouvoir être évaluée, et des observations convergentes émergent des rapports publiés jusqu'ici ${ }^{6}$. Pour les musées, la question critique est par exemple celle de la balance entre leur mission publique et leurs sources de financement, parmi lesquelles les activités commerciales, l'importance et le rayonnement de l'établissement, la nature et le volume de ses collections, ses publics, son influence sur le territoire jouent un rôle crucial dans la nécessaire élaboration d'une stratégie de diffusion de ses images. Mais les politiques de libre réutilisation mises en œuvre par de grands musées américains ou européens, de plus 
en plus nombreux, font aussi émerger, selon les cas, de nouvelles options de financement, de mécénat ou de commercialisation, et de manière plus essentielle, une nouvelle interaction avec les publics, dont il devient possible de connaître les goûts, les curiosités, les modes de communication et les usages créatifs. La gratuité, dans tous ces cas, loin d'être une illusion, devient au contraire un révélateur de la polyvalence extrême des images et de leur caractère agissant en faveur des ouvres qu'elles représentent.

Mais qu'en est-il de ce public aux besoins et aux objectifs spécifiques que constitue la communauté internationale des historiens de l'art ? Autrefois isolée dans sa quête permanente d'images, elle bénéficie aujourd'hui des avancées suscitées par le contexte socio-digital ; avancées qui ne la concernent cependant que partiellement, car la recherche et l'utilisation des images à des fins de recherche ou de publication reste la plupart du temps, étant donné l'hétérogénéité et la fréquente opacité des régimes en place, une tâche hasardeuse et chronophage. Les collections en ligne sont de plus en plus nombreuses, et l'on peut certainement miser, puisqu'elles relèvent des nouvelles missions des musées, sur une quasi-exhaustivité dans un futur plus ou moins éloigné. Mais au-delà de la fonction de signalement, voire d'étude, qu'elles peuvent satisfaire en fonction des possibilités d'exploration de leurs images, force est de constater qu'un nombre non négligeable d'entre elles sont soumises à un régime de copyright, qu'il s'agisse de celui de l'institution ou de celui du photographe, et ce même lorsque les œuvres appartiennent au domaine public. Les musées en cela, particulièrement en France, se distinguent encore des archives et des bibliothèques, qui ont entamé depuis plusieurs années la marche vers le libre accès et la libre réutilisation de leurs contenus numérisés.

Il y a plus d'une explication à ce phénomène, qui ne relève pas de la seule hypothèse, séduisante, que la culture de la protection des œuvres qui définit les musées se serait transférée, de manière quasi capillaire, aux représentations immatérielles de ces mêmes œuvres ${ }^{7}$. Quelles qu'en soient les raisons, l'absence en France d'un régime officiel de gratuité pour les usages scientifiques et éducatifs des images pèse lourdement sur l'histoire de l'art, en l'enfermant dans des pratiques contraintes et dans une temporalité devenues étrangères à son époque. "The burden of images ", le fardeau des images, pour reprendre l'expression paradoxale de Peter Tomlin, dresse en effet une formidable barrière à la fluidité de la recherche et aux circulations qui la constituent ${ }^{8}$. Or l'appel des technologies est devenu pressant : le web, par sa nature même, est un espace de facilitation sans précédent pour toutes les pratiques liées aux images, et particulièrement pour celles qui concernent l'historien d'art : récolte, indexation, description, annotation, manipulation, comparaison, échange. Les collections personnelles, les projets, les archives d'historiens de l'art peuvent devenir des corpus d'images critiques qui s'enrichissent de l'instantanéité de la réflexion, individuelle ou collective, tout en protégeant la paternité des apports. Les images en haute définition partagées par des projets comme ceux qui utilisent le protocole IIIF (International Image Interoperability Framework ${ }^{9}$ ) proviennent $d^{\prime}$ ' entrepôts "institutionnels qui les ont mises à disposition de la communauté via des interfaces de programmation spécifiques : elles sont consultables et manipulables sous des applications compatibles et peuvent ainsi alimenter différents projets de recherche collectifs sur le web. Gratuites et équipées pour l'étude et la publication digitale, elles ont, à travers leur potentiel d'utilisation, à la fois une valeur d'usage et une forte valeur de partage - qui est toutefois, on le voit, différente de celles que portent les images publiées en libre accès pour un large public. Leur avènement récent, tout comme le rapide développement des techniques computationnelles d'analyse, laisse présager des mutations susceptibles de faire considérablement évoluer l'histoire de l'art et du patrimoine dans les années à venir : ainsi, le jour où les archives du connoisseurship seront accessibles, indexées, interopérables, le jour où l'on enseignera au moyen de reproductions d'œuvres en haute définition que les étudiants pourront voir et manipuler eux-mêmes, ou encore, celui ou la recherche d'images sera effectuée par des algorithmes paramétrés sur les pratiques des chercheurs ${ }^{10}$. 
Le fossé s'élargit ainsi de jour en jour entre ces images dotées d'une temporalité sociale, de fonctionnalités dédiées au savoir, et celles qui demeurent protégées, onéreuses, difficiles à obtenir, d'un usage restreint, ou enfermées dans des silos. Parce que les images, au-delà de leur statut d'objet de contemplation et d'analyse, sont devenues des outils visuels polyvalents, une part essentielle du futur des musées, du patrimoine et de la recherche se joue à présent dans la juste reconnaissance de leur valeur ${ }^{11}$. 
1. Peter Weibel, "Le pouvoir des images : des médias visuels aux médias sociaux ", dans Perspective : actualité en histoire de l'art, $\mathrm{n}^{\circ}$ 2012-1, p. 5-7 [en ligne, URL : https://journals.openedition.org/perspective/406 (consulté le 7 juin 2018)].

2. Nous parlons essentiellement ici des reproductions numériques d'œuvres appartenant au domaine public.

3. On trouve de nombreux exemples dans le bilan « Ce qu'il ne fallait pas rater en 2017 autour des projets en Humanités numériques et GLAM », par Antoine Courtin [en ligne, URL : https://goo.gl/cdxAo5 (consulté le 7 juin 2018)].

4. "What The Met shows with its open access program is that it doesn't limit its definition of mission-supporting purposes to scholarly use. The program challenges, and effectively changes, who gets to engage with cultural heritage by placing it where the users are. The investment goes beyond creating access to crafting partnerships with popular platforms for cultural enjoyment and learning like Google Cultural Institute, Pinterest, and Wikipedia. Platforms like these have become hubs for online information seeking and collective knowledge sharing between millions of people. They are dependent on shareable images. Being present here is a way to ensure that the collections of The Met become visible, reachable, and thereby relevant to a far larger part of the world's population than ever before », Merete Sanderhoff, « Open Access Can Never Be Bad News », SMK Open, publié le 6 mars 2017 [en ligne, URL : https://medium.com/smk-open/open-access-can-never-be-bad-news-d33336aad382 (consulté le 31 janvier 2018)].

5. Le passage en Open Access sous licence CCO des images du Metropolitan Museum of Art annoncé par son directeur, Thomas Campbell, en février 2017 (voir https://www.metmuseum.org/press/news/2017/open-access), a été très largement relayé au niveau international par les médias numériques ou classiques.

6. Striking the Balance. How NDMC Members are Balancing Public Access and Commercial Reuse of Digital Content. A report by the Collections Trust commissioned by the NDMC, September 2015, p. 31-32; Effie Kapsalis, The Impact of Open Access on Galleries, Libraries, Museums and Archives, Smithsonian Emerging Leaders Development Program, Avril 2016 [en ligne, URL : https://siarchives.si.edu/sites/default/files/pdfs/2016_03_10_OpenCollections_Public. pdf (consulté le 7 juin 2018)].

7. Christine Kuan, " Maximum Museum. Digital Images, Licensing, and the Future of Museums », contribution dans le cadre de l'American Association of Museums Annual Meeting, Minneapolis, MN, 2 mai 2012 [en ligne, URL : https://www.academia.edu/1548900/Maximum_Museum_Digital_Images_Licensing_and_the_Future_of_ Museums (consulté le 7 juin 2018)], p. 2 : « Museums function, of course, as gatekeepers [...] to objects in physical spaces, which is in many respects an important way of protecting and preserving the collections. Not surprisingly, this gatekeeping mentality has been transplanted to managing digital images. "

8. Peter Tomlin, "Every Man His Book? An Introduction to Open Access in the Arts ", dans Art Documentation: Journal of the Art Libraries Society of North America, vol. 30, n 1, Spring 2011, p. 4-11.

9. Voir http://doc.biblissima-condorcet.fr/iiif-api-presentation.

10. Voir le projet Replica du DHALAB de Lausanne (https://dhlab.epfl.ch/page-128334-en.html), récemment présenté dans le cadre des Lundis numériques de l'Institut national d'histoire de l'art.

11. Il est réjouissant, même si cela ôte un peu à ce texte, de constater qu'entre sa remise et sa publication, le statut des images comme agents de préservation des œuvres et de diffusion de leur connaissance a pris de l'importance, au plan international comme en France. Voir notamment les réalisations du projet ReACH (Reproduction of Art and Cultural Heritage), lancé par l'UNESCO en 2017 et porté par le Victoria and Albert Museum, dont le manifeste Copy Culture: Sharing in the Age of Digital Reproduction, dirigé par Brendan Cormier, pose en prémisse : "For cultural institutions that hold collections for the benefit of the public, the opportunity to provide open access now or in the future to Works in a digital format is an exciting new frontier in their mission to preserve and transmit knowledge, culture and history for present and future generations "(en ligne, URL : https://www.vam.ac.uk/research/projects/ reach-reproduction-of-art-and-cultural-heritage\#outputs (consulté le 25 juin 2018)]. 